\title{
INVARIANT HYPERSURFACES OF ENDOMORPHISMS OF THE PROJECTIVE 3-SPACE
}

\author{
DE-QI ZHANG
}

\begin{abstract}
We consider surjective endomorphisms $f$ of degree $>1$ on the projective $n$-space $\mathbb{P}^{n}$ with $n=3$, and $f^{-1}$-stable hypersurfaces $V$. We show that $V$ is a hyperplane (i.e., $\operatorname{deg}(V)=1$ ) but with four possible exceptions; it is conjectured that $\operatorname{deg}(V)=1$ for any $n \geq 2$; cf. [7, [3].
\end{abstract}

Dedicated to Prof. Miyanishi on the occasion of his 70th birthday

\section{INTRODUCTION}

We work over the field $\mathbb{C}$ of complex numbers. In this paper, we study properties of $f^{-1}$-stable prime divisors of $X$ for endomorphisms $f: \mathbb{P}^{3} \rightarrow \mathbb{P}^{3}$. Below is our main result.

Theorem 1.1. Let $f: \mathbb{P}^{3} \rightarrow \mathbb{P}^{3}$ be an endomorphism of degree $>1$ and $V$ an irreducible hypersurface such that $f^{-1}(V)=V$. Then either $\operatorname{deg}(V)=1$, i.e., $V$ is a hyperplane, or $V$ equals one of the four cubic hypersurfaces $V_{i}=\left\{S_{i}=0\right\}$, where $S_{i}$ 's are as follows, with suitable projective coordinates:

(1) $S_{1}=X_{3}^{3}+X_{0} X_{1} X_{2}$;

(2) $S_{2}=X_{0}^{2} X_{3}+X_{0} X_{1}^{2}+X_{2}^{3}$;

(3) $S_{3}=X_{0}^{2} X_{2}+X_{1}^{2} X_{3}$;

(4) $S_{4}=X_{0} X_{1} X_{2}+X_{0}^{2} X_{3}+X_{1}^{3}$.

We are unable to rule out the four cases in Theorem 1.1 and do not know whether there is any endomorphism $f_{V_{i}}: V_{i} \rightarrow V_{i}$ of $\operatorname{deg}\left(f_{V_{i}}\right)>1$ for $i=2,3$ or 4 , but see Examples 1.5 (for $V_{1}$ ) and 1.2 below.

Example 1.2. There are many endomorphisms $f_{V^{\prime}}: V^{\prime} \rightarrow V^{\prime}$ of $\operatorname{deg}\left(f_{V^{\prime}}\right)>1$ for the normalization $V^{\prime}$ of $V=V_{i}(i=3,4)$, where $V^{\prime} \simeq \mathbb{F}_{1}$ in either case (cf. Remark1.3 below). Conjecture 1.4 below asserts that $f_{V^{\prime}}$ is not lifted from any endomorphism $f: \mathbb{P}^{3} \rightarrow$ $\mathbb{P}^{3}$ restricted to the non-normal cubic surface $V$. Indeed, consider the endomorphism $f_{\mathbb{P}^{2}}: \mathbb{P}^{2} \rightarrow \mathbb{P}^{2}\left(\left[X_{0}, X_{1}, X_{2}\right] \rightarrow\left[X_{0}^{q}, X_{1}^{q}, X_{2}^{q}\right]\right)$ with $q \geq 2$. It lifts to an endomorphism

2000 Mathematics Subject Classification. 37F10, 32H50, 14E20, 14J45.

Key words and phrases. endomorphism, iteration, projective 3-space.

The author is supported by an ARF of NUS. 
$f_{\mathbb{F}_{1}}: \mathbb{F}_{1} \rightarrow \mathbb{F}_{1}$ of $\operatorname{deg}\left(f_{\mathbb{F}_{1}}\right)=q^{2}$, where $\mathbb{F}_{1} \rightarrow \mathbb{P}^{2}$ is the blowup of the point $[0,0,1]$ fixed by $f_{\mathbb{P}^{2}}^{-1}$.

Remark 1.3. Below are some remarks about Theorem 1.1 .

(1) The non-normal locus of $V_{i}(i=3,4)$ is a single line $C$ and stabilized by $f^{-1}$. Let $\sigma: V_{i}^{\prime} \rightarrow V_{i}(i=3,4)$ be the normalization. Then $V_{i}^{\prime}$ is the (smooth) Hirzebruch surface $\mathbb{F}_{1}$ (i.e., the one-point blowup of $\mathbb{P}^{2}$; see [1, Theorem 1.5], [16]) with the conductor $\sigma^{-1}(C) \subset V_{i}^{\prime}$ a smooth section at infinity (for $V_{3}$ ), and the union of the negative section and a fibre (for $V_{4}$ ), respectively. $f \mid V_{i}$ lifts to a (polarized) endomorphism $f_{V_{i}^{\prime}}: V_{i}^{\prime} \rightarrow V_{i}^{\prime}$.

(2) $V_{1}$ (resp. $V_{2}$ ) is unique as a normal cubic (or degree three del Pezzo) surface of Picard number one and with the singular locus Sing $V_{1}=3 A_{2}$ (resp. Sing $V_{2}=$ $\left.E_{6}\right)$; see [17, Theorem 1.2], and [9, Theorem 4.4] for the anti-canonical embedding of $V_{i}$ in $\mathbb{P}^{3}$. $V_{1}$ contains exactly three lines of triangle-shaped whose three vertices form the singular locus of $V_{1}$. And $V_{2}$ contains a single line on which lies its unique singular point. $f^{-3}$ fixes the singular point(s) of $V_{i}(i=1,2)$.

(3) $f^{-1}$ (or its positive power) does not stabilize the only line $L$ on $V_{2}$ by using [14, Theorem 4.3.1] since the pair $\left(V_{2}, L\right)$ is not log canonical at the singular point of $V_{2}$. For $V_{1}$, we do not know whether $f^{-1}$ (or its power) stabilizes the three lines.

1.4. A motivating conjecture. Here are some motivations for our paper. It is conjectured that every hypersurface $V \subset \mathbb{P}^{n}$ stabilized by the inverse $f^{-1}$ of an endomorphism $f: \mathbb{P}^{n} \rightarrow \mathbb{P}^{n}$ of $\operatorname{deg}(f)>1$, is linear. This conjecture is still open when $n \geq 3$ and $V$ is singular, since the proof of [3] is incomplete as we were informed by an author. The smooth hypersurface case was settled in the affirmative in any dimension by Cerveau Lins Neto [4] and independently by Beauville [2]. See also [15, Theorem 1.5 in arXiv version], [18] and [19] for related results.

By Theorem 1.1, this conjecture is true when $n=3$ but with four exceptional cubic surfaces $V_{i}$ which we could not rule out.

From the dynamics point of view, as seen in Dinh-Sibony [5, Theorem 1.3, Corollary 1.4], $f: \mathbb{P}^{n} \rightarrow \mathbb{P}^{n}$ behaves nicely exactly outside those $f^{-1}$-stabilized subvarieties. We refer to Fornaess-Sibony [7], and [5] for further references.

A smooth hypersurface $X$ in $\mathbb{P}^{n+1}$ with $\operatorname{deg}(X) \geq 3$ and $n \geq 2$, has no endomorphism $f_{X}: X \rightarrow X$ of degree $>1$ (cf. [4], [2, Theorem]). However, singular $X$ may have plenty of endomorphisms $f_{X}$ of arbitrary degrees as shown in Example 1.5 below. Conjecture 1.4 asserts that such $f_{X}$ can not be extended to an endomorphism of $\mathbb{P}^{n+1}$. 
Example 1.5. We now construct many polarized endomorphisms for some degree $n+1$ hypersurface $X \subset \mathbb{P}^{n+1}$, with $X$ isomorphic to the $V_{1}$ in Theorem 1.1 when $n=2$. Let

$$
f=\left(F_{0}, \ldots, F_{n}\right): \mathbb{P}^{n} \rightarrow \mathbb{P}^{n}
$$

$(n \geq 2)$, with $F_{i}=F_{i}\left(X_{0}, \ldots, X_{n}\right)$ homogeneous, be any endomorphism of degree $q^{n}>1$, such that $f^{-1}(S)=S$ for a reduced degree $n+1$ hypersurface $S=\left\{S\left(X_{0}, \ldots, X_{n}\right)=0\right\}$. So $S$ must be normal crossing and linear: $S=\sum_{i=0}^{n} S_{i}$ (cf. [15, Theorem 1.5 in arXiv version]). Thus we may assume that $f=\left(X_{0}^{q}, \ldots, X_{n}^{q}\right)$ and $S_{i}=\left\{X_{i}=0\right\}$. The relation $S \sim(n+1) H$ with $H \subset \mathbb{P}^{n}$ a hyperplane, defines

$$
\pi: X=\operatorname{Spec} \oplus_{i=0}^{n} \mathcal{O}(-i H) \rightarrow \mathbb{P}^{n}
$$

which is a Galois $\mathbb{Z} /(n+1)$-cover branched over $S$ so that $\pi^{*} S_{i}=(n+1) T_{i}$ with the restriction $\pi \mid T_{i}: T_{i} \rightarrow S_{i}$ an isomorphism.

This $X$ is identifiable with the degree $n+1$ hypersurface

$$
\left\{Z^{n+1}=S\left(X_{0}, \ldots, X_{n}\right)\right\} \subset \mathbb{P}^{n+1}
$$

and has singularity of type $z^{n+1}=x y$ over the intersection points of $S$ locally defined as $x y=0$. Thus, when $n=2$, we have $\operatorname{Sing} X=3 A_{2}$ and $X$ is isomorphic to the $V_{1}$ in Theorem 1.1 (cf. Remark 1.3). We may assume that

$$
f^{*} S\left(X_{0}, \ldots, X_{n}\right)=S\left(X_{0}, \ldots, X_{n}\right)^{q}
$$

after replacing $S\left(X_{0}, \ldots, X_{n}\right)$ by a scalar multiple, so $f$ lifts to an endomorphism

$$
g=\left(Z^{q}, F_{0}, \ldots, F_{n}\right)
$$

of $\mathbb{P}^{n+1}$ (with homogeneous coordinates $\left[Z, X_{0}, \ldots, X_{n}\right]$ ), stabilizing $X$, so that

$$
g_{X}:=g \mid X: X \rightarrow X
$$

is a polarized endomorphism of $\operatorname{deg}\left(g_{X}\right)=q^{n}$ (cf. [15, Lemma 2.1]). Note that $g^{-1}(X)$ is the union of $q$ distinct hypersurfaces

$$
\left\{Z=\zeta^{i} S\left(X_{0}, \ldots, X_{n}\right)\right\} \subset \mathbb{P}^{n+1}
$$

(all isomorphic to $X$ ), where $\zeta:=\exp (2 \pi \sqrt{-1} / q)$.

This $X$ has only Kawamata log terminal singularities and $\operatorname{Pic} X=\left(\operatorname{Pic} \mathbb{P}^{n+1}\right) \mid X(n \geq$ 2 ) is of rank one, using Lefschetz type theorem [12, Example 3.1.25] when $n \geq 3$. We have $f^{-1}\left(S_{i}\right)=S_{i}$ and $g_{X}^{-1}\left(T_{i}\right)=T_{i}$, where $0 \leq i \leq n$.

When $n=2$, the relation $(n+1)\left(T_{1}-T_{0}\right) \sim 0$ gives rise to an étale-in-codimenion-one $\mathbb{Z} /(n+1)$-cover

$$
\tau: \mathbb{P}^{n} \simeq \widetilde{X} \rightarrow X
$$


so that $\sum_{i=0}^{n} \tau^{*} T_{i}$ is a union of $n+1$ normal crossing hyperplanes; indeed, $\tau$ restricted over $X \backslash \operatorname{Sing} X$, is its universal cover (cf. [13, Lemma 6]), so that $g_{X}$ lifts up to $\widetilde{X}$. A similar result seems to be true for $n \geq 3$, by considering the 'composite' of the $\mathbb{Z} /(n+1)$-covers given by $(n+1)\left(T_{i}-T_{0}\right) \sim 0(1 \leq i<n)$.

\section{Proofs of Theorem 1.1 and Remark 1.3}

We use the standard notation in Hartshorne's book and [11].

2.1. We now prove Theorem 1.1 and Remark 1.3, By [15, Theorem 1.5 in arXiv version], we may assume that $V \subset \mathbb{P}^{3}$ is an irreducible rational singular cubic hypersurface.

We first consider the case where $V$ is non-normal. Such $V$ is classified in [6, Theorem 9.2.1] to the effect that either $V=V_{i}(i=3,4)$ or $V$ is a cone over a nodal or cuspidal rational planar cubic curve $B$. The description in Remark 1.3 on $V_{3}, V_{4}$ and their normalizations, is given in [16, Theorem 1.1], [1, Theorem 1.5, Case (C), (E1)]; the $f^{-1}$-invariance of the non-normal locus $C$ is proved in [15, Proposition 5.4 in arXiv version].

We consider and will rule out the case where $V$ is a cone over $B$. Since $V$ is normal crossing in codimension 1 (cf. [15, Theorem 1.5 or Proposition 5.4 in arXiv version]), the base $B$ of the cone $V$ is nodal. Let $P$ be the vertex of the cone $V$, and $L \subset V$ the generating line lying over the node of $B$. Then $f_{V}:=f \mid V$ satisfies the assertion that $f_{V}^{-1}(P)=P$. Indeed, the normalization $V^{\prime}$ of $V$ is a cone over a smooth rational (twisted) cubic curve (in $\mathbb{P}^{3}$ ), i.e., the contraction of the $(-3)$-curve on the Hirzebruch surface $\mathbb{F}_{3}$ of degree $3 ; f_{V}$ lifts to an endomorphism $f_{V^{\prime}}$ of $V^{\prime}$ so that the conductor $C^{\prime} \subset V^{\prime}$ is preserved by $f_{V^{\prime}}^{-1}$ (cf. [15, Proposition 5.4 in arXiv version]) and consists of two distinct generating lines $L_{i}$ (lying over $L$ ). Thus $f_{V^{\prime}}^{-1}$ fixes the vertex $L_{1} \cap L_{2}$ (lying over $P$ ). Hence $f_{V}^{-1}(P)=P$ as asserted.

By [15, Lemma 5.9 in arXiv version], $f: \mathbb{P}^{3} \rightarrow \mathbb{P}^{3}$ (with $\operatorname{deg}(f)=q^{3}>1$ say) descends, via the projection $\mathbb{P}^{3} \cdots \rightarrow \mathbb{P}^{2}$ from the point $P$, to an endomorphism $h: \mathbb{P}^{2} \rightarrow \mathbb{P}^{2}$ with $\operatorname{deg}(h)=q^{2}>1$ so that $h^{-1}(B)=B$. This and $\operatorname{deg}(B)=3>1$ contradict the linearity property of $h^{-1}$-stable curves in $\mathbb{P}^{2}$ (see e.g. Theorem 1.5 and the references in [15, arXiv version]).

2.2. Next we consider the case where $V \subset \mathbb{P}^{3}$ is a normal rational singular cubic hypersurface. By the adjunction formula,

$$
-K_{V}=-\left(K_{\mathbb{P}^{3}}+V\right)|V \sim H| V
$$


which is ample, where $H \subset \mathbb{P}^{3}$ is a hyperplane. Since $K_{V}$ is a Cartier divisor, $V$ has only $\mathrm{Du}$ Val (or rational double, or $A D E$ ) singularities. Let

$$
\sigma: V^{\prime} \rightarrow V
$$

be the minimal resolution. Then

$$
K_{V^{\prime}}=\sigma^{*} K_{V} \sim \sigma^{*}(-H \mid V)
$$

For $f: \mathbb{P}^{3} \rightarrow \mathbb{P}^{3}$, we can apply the result below to $f_{V}:=f \mid V$.

Lemma 2.3. Let $V \subset \mathbb{P}^{3}$ be a normal cubic surface, and $f_{V}: V \rightarrow V$ an endomorphism such that $f_{V}^{*}(H \mid V) \sim q H \mid V$ for some $q>1$ and the hyperplane $H \subset \mathbb{P}^{3}$. Let

$$
S(V)=\left\{G \subset V \mid G: \text { irreducible, } G^{2}<0\right\}
$$

be the set of negative curves on $V$, and set

$$
E_{V}:=\sum_{E \in S(V)} E
$$

Replacing $f_{V}$ by its positive power, we have:

(1) If $f_{V}^{*} G \equiv a G$ for some Weil divisor $G \not \equiv 0$, then $a=q$. We have

$$
f_{V}^{*}(L \mid V) \sim q(L \mid V)
$$

for every divisor $L$ on $\mathbb{P}^{3}$. Especially, $\operatorname{deg}\left(f_{V}\right)=q^{2} ; K_{V} \sim-H \mid V$ satisfies $f_{V}^{*} K_{V} \sim q K_{V}$.

(2) $S(V)$ is a finite set. $f_{V}^{*} E=q E$ for every $E \in S(V)$. So $f_{V}^{*} E_{V}=q E_{V}$.

(3) A curve $E \subset V$ is a line in $\mathbb{P}^{3}$ if and only if $E$ is equal to $\sigma\left(E^{\prime}\right)$ for some $(-1)$ curve $E^{\prime} \subset V^{\prime}$.

(4) Every curve $E \in S(V)$ is a line in $\mathbb{P}^{3}$.

(5) We have

$$
K_{V}+E_{V}=f_{V}^{*}\left(K_{V}+E_{V}\right)+\Delta
$$

for some effective divisor $\Delta$ containing no line in $S(V)$, so that the ramification divisor

$$
R_{f_{V}}=(q-1) E_{V}+\Delta .
$$

In particular, the cardinality $\# S(V) \leq 3$, and the equality holds exactly when $K_{V}+E_{V} \sim_{\mathbb{Q}} 0$; in this case, $f_{V}$ is étale outside the three lines of $S(V)$ and $f_{V}^{-1}(\operatorname{Sing} V)$. 
Proof. For (1) and (2), we refer to [15, Lemma 2.1] and [14, Proposition 3.6.8] and note that $L \sim b H$ for some integer $b$.

(3) We may assume that $E^{\prime} \simeq \mathbb{P}^{1}$, where $E^{\prime}:=\sigma^{\prime}(E)$ is the proper transform of $E$. (3) is true because $E$ is a line if and only if

$$
1=E \cdot H \mid V\left(=E^{\prime} \cdot \sigma^{*}(H \mid V)=E^{\prime} \cdot\left(-K_{V^{\prime}}\right)\right),
$$

and by the genus formula $-2=2 g\left(E^{\prime}\right)-2=\left(E^{\prime}\right)^{2}+E^{\prime} \cdot K_{V^{\prime}}$.

(4) $E^{\prime}:=\sigma^{\prime}(E)$ satisfies $E^{\prime} \cdot K_{V^{\prime}}=E \cdot K_{V}<0$ and $\left(E^{\prime}\right)^{2} \leq E^{\prime} \cdot \sigma^{*} E=E^{2}<0$. Hence $E^{\prime}$ is a (-1)-curve by the genus formula. Thus (4) follows from (3).

(5) The first part is true because, by (2), the ramification divisor $R_{f_{V}}=(q-1) E_{V}+$ (other effective divisors). Also, by (1) and (2), $\Delta \sim(1-q)\left(K_{V}+E_{V}\right)$. Since $K_{V} \cdot E=-1$ for every $E \in S(V)$ (by (4)), we have

$$
0 \leq-K_{V} \cdot \Delta=-K_{V} \cdot(1-q)\left(K_{V}+E_{V}\right)=(q-1)(3-\# S(V)) .
$$

Now the second part of (5) follows from this and the fact that $\Delta=0$ if and only if $-K_{V} \cdot \Delta=0$ since $-K_{V}$ is ample. The last part of (5) follows from the purity of branch loci and the description of $R_{f_{V}}$ in (5).

2.4. We now prove Theorem 1.1 and Remark 1.3 for the normal cubic surface $V$. We use the notation in Lemma 2.3. Suppose that the Picard number

$$
\rho:=\rho(V) \geq 3
$$

Since $K_{V}$ is not nef and by the minimal model program for klt surfaces, there is a composite

$$
V=V_{\rho} \stackrel{\tau_{\rho}}{\rightarrow} V_{\rho-1} \cdots \stackrel{\tau_{3}}{\rightarrow} V_{2}
$$

of birational extremal contractions such that

$$
\rho\left(V_{i}\right)=i
$$

Let

$$
E_{i} \subset V_{i}
$$

be the exceptional (irreducible) divisor of $\tau_{i}: V_{i} \rightarrow V_{i-1}$. Since $V$ is Du Val, either $E_{i}$ is contained in the smooth locus $V_{i} \backslash \operatorname{Sing}\left(V_{i}\right)$ and is a $(-1)$-curve, or $E_{i}$ contains exactly one singular point

$$
P_{i} \in \operatorname{Sing} V_{i}
$$

of type $A_{n_{i}}$ so that $\tau_{i}\left(E_{i}\right) \in V_{i-1}$ is a smooth point. In particular, every $V_{i}$ is still Du Val. Let

$$
V_{i}^{\prime} \rightarrow V_{i}
$$


be the minimal resolution. Since $-K_{V_{i}}$ is the pushforward of the ample divisor $-K_{V}$, it is ample. So $V_{i}$ is still a Gorenstein del Pezzo surface. Noting that $K_{V_{i}^{\prime}}$ is the pullback of $K_{V_{i}}$, we have

$$
\left(K_{V_{i-1}^{\prime}}^{2}=\right) K_{V_{i-1}}^{2}=K_{V_{i}}^{2}+\left(n_{i}+1\right) \geq 3+(0+1)=4
$$

for all $3 \leq i \leq \rho$.

Note that the proper transform

$$
E_{i}(V) \subset V
$$

of $E_{i} \subset V_{i}$ is a negative curve. Since $f_{V}^{-1}$ stabilizes every negative curve in $S(V)$ and especially $E_{i}(V)$ (when $f$ is replaced by its positive power, as seen in Lemma 2.3), $f_{V}$ descends to

$$
f_{i}: V_{i} \rightarrow V_{i}
$$

The $V_{2}^{\prime}$ and $S\left(V_{2}^{\prime}\right)$, the set of negative curves on $V_{2}^{\prime}$, are classified in [17, Figure 6]. Since $K_{V_{2}}^{2} \geq 4,\left(V_{2}^{\prime}, S\left(V_{2}^{\prime}\right)\right)$ is as described in one of the last 10 cases in [ibid.]. For example, we write

$$
V_{2}=V_{2}\left(2 A_{2}+A_{1}\right)
$$

if Sing $V_{2}$ consist of two points of type $A_{2}$ and one point of type $A_{1}$.

Except the four cases

$$
V_{2}\left(D_{4}\right), V_{2}\left(4 A_{1}\right), V_{2}\left(A_{3}\right), V_{2}\left(2 A_{1}\right)
$$

in [ibid.], exactly two $(-1)$-curves in $S\left(V_{2}^{\prime}\right)$ map to intersecting negative curves

$$
M_{i} \in S\left(V_{2}\right)
$$

so that

$$
S(V)=\left\{E_{\rho}, M_{1}(V), M_{2}(V)\right\}
$$

with

$$
M_{i}(V) \subset V
$$

the proper transform of $M_{i}$, so

$$
K_{V}+E_{\rho}+M_{1}(V)+M_{2}(V) \sim_{\mathbb{Q}} 0
$$

(cf. Lemma 2.3) and hence $K_{V_{2}}+M_{1}+M_{2} \sim_{\mathbb{Q}} 0$, which is impossible by a simple calculation and blowing down $V_{2}^{\prime}$ to its relative minimal model.

For each of the above four exceptional cases, we may assume that $f_{2}^{-1}$ stabilizes both extremal rays $\mathbb{R}_{+}\left[M_{i}\right]$ of the closed cone $\overline{\mathrm{NE}}\left(V_{2}\right)$ of effective 1 -cycles, with

$$
M_{i} \subset V_{2}
$$


the image of some $(-1)$-curve on $V_{2}^{\prime}$, where both extremal rays are of fibre type in the cases $V_{2}\left(D_{4}\right)$ and $V_{2}\left(4 A_{1}\right)$, where the first (resp. second) is of fibre type (resp. birational type) in the cases $V_{2}\left(A_{3}\right)$ and $V_{2}\left(2 A_{1}\right)$. Let

$$
F_{i}\left(\sim 2 M_{i}\right)
$$

with $i=1,2$, or with $i=1$ only, be the fibre of the extremal fibration

$$
\varphi_{i}=\Phi_{\left|2 M_{i}\right|}: V \rightarrow B_{i} \simeq \mathbb{P}^{1}
$$

passing through the point $\left(\tau_{3} \circ \cdots \circ \tau_{\rho}\right)\left(E_{\rho}\right)$. Then the proper transform

$$
F_{i}(V) \subset V
$$

of $F_{i}$ is a negative curve so that

$$
E_{V}=F_{1}(V)+F_{2}(V)+E_{\rho}
$$

in the cases $V_{2}\left(D_{4}\right)$ and $V_{2}\left(4 A_{1}\right)$, and

$$
E_{V}=F_{1}(V)+M_{2}(V)+E_{\rho}
$$

in the cases $V_{2}\left(A_{3}\right)$ and $V_{2}\left(2 A_{1}\right)$ (cf. Lemma 2.3). Then $K_{V}+E_{V} \sim_{\mathbb{Q}} 0$, and hence $K_{V_{2}}+F_{1}+F_{2} \sim_{\mathbb{Q}} 0$ or $K_{V_{2}}+F_{1}+M_{2} \sim_{\mathbb{Q}} 0$ where the latter is impossible by a simple calculation as in the early paragraph. Thus

$$
K_{V_{2}}+F_{1}+F_{2} \sim_{\mathbb{Q}} 0 .
$$

By making use of Lemma $2.3(1)$ or $(2), f_{2}^{*} F_{i}=q F_{i}$, and $f_{2}$ descends to an endomorphism

$$
f_{B_{1}}: B_{1} \rightarrow B_{1}
$$

of degree $q$. Thus the ramification divisor of $f_{B_{1}}$ is of degree $2(q-1)$ by the Hurwitz formula, and is hence equal to

$$
(q-1) P+\sum\left(b_{i}-1\right) P_{i}
$$

with

$$
\sum\left(b_{i}-1\right)=q-1
$$

where $P \in B_{1}$ so that $F_{1}$ lies over $P$. But then

$$
R_{f_{2}} \geq(q-1)\left(F_{1}+F_{2}\right)+\sum\left(b_{i}-1\right) F_{i}^{\prime}
$$

where $F_{i}^{\prime}$ are fibres of $\varphi_{1}$ lying over $P_{i}$, so that

$$
K_{V_{2}}+F_{1}+F_{2} \geq f_{2}^{*}\left(K_{V_{2}}+F_{1}+F_{2}\right)+\sum\left(b_{i}-1\right) F_{i}^{\prime}
$$

which is impossible since $K_{V_{2}}+F_{1}+F_{2} \sim_{\mathbb{Q}} 0$. 
2.5. Consider the case $\rho(V)=2$. Then the minimal resolution

$$
V^{\prime} \rightarrow V
$$

and its negative curves are described in one of the first five cases in [17, Figure 6].

For the case $V=V\left(A_{5}\right)$, two $(-1)$-curves on $V^{\prime}$ map to two negative curves

$$
M_{1}, M_{2}
$$

on $V$. Note that $f_{V}^{*}\left(M_{i}\right)=q^{*} M_{i}$ (see Lemma 2.3). There is a contraction

$$
V \rightarrow \mathbb{P}^{2}
$$

of $M_{1}$ so that the image of $M_{2}$ is a plane conic preserved by $f_{P}^{-1}$ where

$$
f_{P}: \mathbb{P}^{2} \rightarrow \mathbb{P}^{2}
$$

is the descent of $f_{V}$ (of degree $q^{2}>1$ ), contradicting [15, Theorem 1.5(4) in arXiv version].

For the case $V\left(2 A_{2}+A_{1}\right)$, there are exactly five $(-1)$-curves

$$
M_{i}^{\prime} \subset V^{\prime}
$$

with $M_{i} \subset V$ their images. Moreover, $M_{1}^{\prime} \cdot M_{2}^{\prime}=1$ and both $M_{i}(i=1,2)$ are negative curves on $V$; each $M_{j}^{\prime}(j=3,4)$ meets the isolated (-2)-curve; $M_{1}$ and $M_{3}$ (reap. $M_{2}$ and $M_{4}$ ) meet the same component of one (resp. another) (-2)-chain of type $A_{2}$. We have

$$
M_{1}+M_{2} \sim 2 L
$$

for some integral Weil divisor $L$, by considering a relative minimal model of $V^{\prime}$. In fact, $M_{1}+3 M_{2} \sim 4 M_{3}$. Since $f_{V}^{*}\left(M_{1}+M_{2}\right)=q\left(M_{1}+M_{2}\right)$ (see Lemma 2.3),$f_{V}$ lifts to some

$$
g: U \rightarrow U
$$

Here the double cover (given by the relation $M_{1}+M_{2} \sim 2 L$ )

$$
\pi: U=\operatorname{Spec} \oplus_{i=0}^{1} \mathcal{O}(-i L) \rightarrow V
$$

is branched along $M_{1}+M_{2}$. Indeed, when $2 \nmid q$, the normalization

of the fibre product of $\pi: U \rightarrow V$ and $f_{V}: V \rightarrow V$ is isomorphic to $U$ and we take $g$ to be the first projection $\hat{U} \rightarrow U$; when $2 \mid q$, we have $\hat{U}=V \coprod V$ and let $g$ be the composite of $\pi: U \rightarrow V$, the inclusion $V \cup \emptyset \rightarrow V \coprod V$ and the first projection $\hat{U} \rightarrow U$. Now $\operatorname{Sing} U$ consists of a type $A_{1}$ singularity lying over $M_{1} \cap M_{2}$ and four points in $\pi^{-1}$ (Sing $V$ ) of type $A_{1}, A_{1}, \frac{1}{3}(1,1)$ and $\frac{1}{3}(1,1)$, and every $M_{j}(j=3,4)$ splits into two negative curves on $U$ which are hence preserved by $g^{-1}$ (as in Lemma 2.3 after $f_{V}$ is replaced by its positive power). Thus $f_{V}^{-1}\left(M_{i}\right)=M_{i}(1 \leq i \leq 4)$. As in the proof of Lemma 2.3 , 
$E_{V}^{\prime}:=M_{1}+M_{2}+M_{3}$ satisfies $K_{V}+E_{V}^{\prime} \sim_{\mathbb{Q}} 0$ and $f_{V}$ is étale over $V \backslash\left(E_{V}^{\prime} \cup \operatorname{Sing} V\right)$. The latter contradicts the fact that $f_{V}^{*} M_{4}=q M_{4}$ and hence $f_{V}$ has ramification index $q$ along $M_{4}$.

For $V=V\left(A_{4}+A_{1}\right)$, there are exactly four $(-1)$-curves

$$
M_{i}^{\prime} \subset V^{\prime}
$$

with $M_{i} \subset V$ their images. We may so label that the five $(-2)$-curves and $M_{j}^{\prime}(j=1,2,3)$ form a simple loop:

$$
M_{1}^{\prime}-(-2)-(-2)-(-2)-(-2)-M_{2}^{\prime}-M_{3}^{\prime}-(-2)-M_{1}^{\prime}
$$

both $M_{j}(j=2,3)$ are negative curves on $V$. We can verify that $M_{3}+2 M_{2} \sim 3\left(M_{5}-M_{2}\right)$, and $M_{1}+M_{2} \sim M_{5}$, where $M_{5}$ is the image of a curve $M_{5}^{\prime} \simeq \mathbb{P}^{1}$ and $M_{5}^{\prime}$ is the smooth fibre (passing through the intersection point of $M_{3}^{\prime}$ and the isolated (-2)-curve) of the $\mathbb{P}^{1}$-fibration on $V^{\prime}$ with a singular fibre consisting of $M_{1}^{\prime}, M_{2}^{\prime}$ and the (-2)-chain of type $A_{4}$ sitting in between them. As in the case $V\left(2 A_{2}+A_{1}\right), f_{V}$ lifts to

$$
g: U \rightarrow U
$$

on the triple cover $U$ defined by the relation $M_{3}+2 M_{2} \sim 3\left(M_{5}-M_{2}\right)$, so that each $M_{i}$ $(i=4,5)$ splits into three negative curves on $U$ preserved by $g^{-1}$. Hence $f_{V}^{-1}\left(M_{i}\right)=M_{i}$ $(i=2, \ldots, 5)$. But then for $E_{V}^{\prime}:=M_{2}+M_{3}+M_{4}$ we have $K_{V}+E_{V}^{\prime} \sim_{\mathbb{Q}} 0$ as in the proof of Lemma 2.3 so that $f_{V}$ is étale over $V \backslash\left(E_{V}^{\prime} \cup \operatorname{Sing} V\right)$, contradicting the fact that $f_{V}$ has ramification index $q$ along $M_{5}$.

For $V=V\left(D_{5}\right)$, the lonely $(-1)$-curve $M_{1}^{\prime}$ and the intersecting (-1)-curves $M_{2}^{\prime} \cup M_{3}^{\prime}$ on $V^{\prime}$ satisfy $2 M_{1} \sim M_{2}+M_{3}$ where

$$
M_{i} \subset V
$$

denotes the image of $M_{i}^{\prime}$ (indeed, the three $M_{i}^{\prime}$ together with the five (-2)-curves form the support of two singular fibres and a section in a $\mathbb{P}^{1}$-fibration). As in the case $V\left(2 A_{2}+A_{1}\right)$, $f_{V}$ lifts to

$$
g: U \rightarrow U
$$

on the double cover $U$ defined by the relation $2 M_{1} \sim M_{2}+M_{3}$, so that $M_{1}$ splits into two negative curves on $U$ preserved by $g^{-1}$. Thus $f_{V}^{-1}\left(M_{1}\right)=M_{1}$. Hence $\left(V, M_{1}\right)$ is $\log$ canonical (cf. [14, Theorem 4.3.1]). But $\left(V, M_{1}\right)$ is not log canonical because $M_{1}^{\prime}$ meets the $(-2)$-tree of type- $D_{5}$ in a manner different from the classification of $[10$, Theorem 9.6]. We reach a contradiction.

For $V=V\left(A_{3}+2 A_{1}\right)$, let $M_{1}^{\prime} \subset V^{\prime}$ be the $(-1)$-curve meeting the middle component of the (-2)-chain of type $A_{3}$, let $M_{3}^{\prime}$ be the $(-1)$-curve meeting two isolated (-2)-curves, 
and let $M_{2}^{\prime}$ be the $(-1)$-curve meeting both $M_{1}^{\prime}$ and $M_{3}^{\prime}$. Then the images

$$
M_{i} \subset V
$$

of $M_{i}^{\prime}$ satisfy $2 M_{1} \sim 2 M_{3}$ (indeed, $M_{1}^{\prime}, M_{3}^{\prime}$ and the five (-2)-curves form the support of two singular fibres in some $\mathbb{P}^{1}$-fibration). The relation $2\left(M_{1}-M_{3}\right) \sim 0$ defines a double cover

$$
\pi: U=\operatorname{Spec} \oplus_{i=0}^{1} \mathcal{O}\left(-i\left(M_{1}-M_{3}\right)\right) \rightarrow V
$$

étale over $V \backslash \operatorname{Sing} V$. In fact, $\pi$ restricted over $V \backslash \operatorname{Sing} V$, is the universal cover over it, so $U$ is again a Gorenstein del Pezzo surface and hence the irregularity $q(U)=0$. Thus $f_{V}$ lifts to

$$
g: U \rightarrow U
$$

Now $\pi^{-1}$ (Sing $V$ ) consists of two smooth points and the unique singular point of $U$ (of type $\left.A_{1}\right)$, and each $\pi^{*} M_{i}(i=1,2)$ splits into two negative curves $M_{i}(1), M_{i}(2)$ on $U$ preserved by $g^{-1}$; thus $f_{V}^{*} M_{i}=q M_{i}$ and $g^{*} M_{i}(j)=q M_{i}(j)$ (as in Lemma $2.3(1)$ ).

We assert that $f_{V}^{-1}$ permutes members of the pencil

$$
\Lambda:=\left|M_{1}+M_{2}\right|
$$

It suffices to show that $g^{-1}$ permutes members of the irreducible pencil

$$
\Lambda_{U}
$$

(parametrized by $\mathbb{P}^{1}$ for $q(U)=0$ ) which is the pullback of $\Lambda$. Now $\pi^{*}\left(M_{1}+M_{2}\right)$ splits into two members

$$
M_{1}(j)+M_{2}(j)=\operatorname{div}\left(\xi_{j}\right)
$$

(in local equation; $j=1,2)$ which are preserved by $g^{-1}$ and span $\Lambda_{U}$. We may assume that $g^{*} \xi_{j}=\xi_{j}^{q}$ after replacing the equation by a scalar multiple. Then the $g^{*}$-pullback of every member $\operatorname{div}\left(a \xi_{1}+b \xi_{2}\right)$ in $\Lambda_{U}$ is equal to $\operatorname{div}\left(a \xi_{1}^{q}+b \xi_{2}^{q}\right)$ and hence is the union of members in $\Lambda_{U}$ because we can factorize $a \xi_{1}^{q}+b \xi_{2}^{q}$ as a product of linear forms in $\xi_{1}, \xi_{2}$. This proves the assertion.

By the assertion and since $f_{V}^{*}\left(M_{1}+M_{2}\right)=q\left(M_{1}+M_{2}\right), f_{V}$ descends to an endomorphism

$$
f_{B}: B \rightarrow B
$$

of degree $q$ on the curve $B \simeq \mathbb{P}^{1}$ parametrizing the pencil $\Lambda$. We have $f_{B}^{*} P_{0}=q P_{0}$ for the point parametrizing the member $M_{1}+M_{2}$ of $\Lambda$. Write $K_{B}=f_{B}^{*} K_{B}+R_{f_{B}}$, where the ramification divisor

$$
R_{f_{B}}=(q-1) P_{0}+\Delta_{B}
$$

with $\Delta_{B}=\sum\left(b_{i}-1\right) Q_{j}$ of degree $q-1$ for some $b_{i} \geq 2$. Thus the ramification divisor

$$
R_{f_{V}}=(q-1)\left(M_{1}+M_{2}\right)+\Delta_{V}
$$


with $\Delta_{V}=\sum\left(b_{i}-1\right) F_{i}+$ (other effective divisor), where

$$
F_{i} \in \Lambda
$$

is parametrized by $Q_{i}$. On the other hand, one can verify that $-K_{V} \sim 2 M_{1}+M_{2}$, by blowing down to a relative minimal model of $V^{\prime}$; indeed, $M_{2}$ is a double section of the $\mathbb{P}^{1}$-fibration

$$
\varphi:=\Phi_{\left|2 M_{1}\right|}: V \rightarrow \mathbb{P}^{1}
$$

So $-M_{1} \sim K_{V}+M_{1}+M_{2}=f_{V}^{*}\left(K_{V}+M_{1}+M_{2}\right)+\Delta_{V}$ and hence, by Lemma 2.3 (1),

$$
\left(b_{1}-1\right) F_{1} \leq \Delta_{V} \sim(1-q)\left(K_{V}+M_{1}+M_{2}\right) \sim(q-1) M_{1} .
$$

This is impossible because $F_{1}$ is horizontal to the half fibre $M_{1}$ of $\varphi$. Indeed, $F_{1} \cdot M_{1}=$ $\left(M_{1}+M_{2}\right) \cdot M_{1}=M_{2} \cdot M_{1}=1$.

2.6. Consider the last case $\rho(V)=1$. Since $K_{V}^{2}=3$, we have

$$
V=V\left(3 A_{2}\right), V\left(E_{6}\right), \text { or } V\left(A_{1}+A_{5}\right),
$$

and the minimal resolution

$$
V^{\prime} \rightarrow V
$$

and the negative curves on $V^{\prime}$ are described in [17, Figure 5]. For the first two cases, $V$ is isomorphic to $V_{i}(i=1$, or 2$)$ in Theorem 1.1 by the uniqueness result in [17, Theorem 1.2] and by [9, Theorem 4.4].

For $V=V\left(A_{1}+A_{5}\right)$, the images

$$
M_{i} \subset V
$$

of the two $(-1)$-curves $M_{i}^{\prime} \subset V^{\prime}$ satisfy $2 M_{1} \sim 2 M_{2}$; indeed, $M_{i}^{\prime}$ together with the six $(-2)$-curves form the support of two singular fibres and a section in some $\mathbb{P}^{1}$-fibration. Let

$$
\pi: U \rightarrow V
$$

be the double cover given by the relation $2\left(M_{1}-M_{2}\right) \sim 0$. In fact, $\pi$ restricted over $V \backslash \operatorname{Sing} V$, is the universal cover over it. So $f_{V}$ lifts to

$$
g: U \rightarrow U
$$

As in the case $V\left(A_{3}+2 A_{1}\right)$, if we let $M_{1}^{\prime}$ be the one meeting the second component of the (-2)-chain of type $A_{5}$, then $\pi^{*} M_{1}$ splits into two negative curves on $U$ preserved by $g^{-1}$. Thus $f_{V}^{-1}\left(M_{1}\right)=M_{1}$, and, as in the case $V\left(D_{5}\right)$ above, contradicts [14, Theorem 4.3.1] and [10, Theorem 9.6].

This completes the proof of Theorem 1.1 for normal cubic surfaces and hence the whole of Theorem 1.1. To determine the equations of $V_{i}(i=1,2)$, we can check that the equations in Theorem 1.1 possess the right combination of singularities and then use 
the very ampleness of $-K_{V_{i}}$ to embed $V_{i}$ in $\mathbb{P}^{3}$ as in [9] and the uniqueness of $V_{i}$ up to isomorphism, and hence up to projective transformation by [9] (cf. [17, Theorem 1.2]).

2.7. Now we prove Remark 1.3. From Lemma 2.3 till now, we did not assume the hypothesis $(*)$ that $f_{V}$ is the restriction of some $f: \mathbb{P}^{3} \rightarrow \mathbb{P}^{3}$ whose inverse stabilizes $V$. From now on till the end of the paper, we assume this hypothesis $(*)$.

For $V=V\left(E_{6}\right)$ or $V\left(3 A_{2}\right)$, the relation $V \sim 3 H$ defines a triple cover

$$
\pi: X=\operatorname{Spec} \oplus_{i=0}^{2} \mathcal{O}(-i H) \rightarrow V
$$

branched along $V$. Then

$$
X=\left\{Z^{3}=V\left(X_{0}, \ldots, X_{3}\right)\right\} \subset \mathbb{P}^{4}
$$

is a cubic hypersurface, where we let $V\left(X_{0}, \ldots, V_{3}\right)$ be the cubic form defining $V \subset \mathbb{P}^{3}$. Our $\pi^{-1}$ restricts to a bijection $\pi^{-1}: \operatorname{Sing} V \rightarrow \operatorname{Sing} X$. As in Example 1.5, $f$ lifts to $\left(Z^{q}, f\right): \mathbb{P}^{4} \rightarrow \mathbb{P}^{4}$ stabilizing $X$, so that the restriction

$$
g=\left(Z^{q}, f\right) \mid X: X \rightarrow X
$$

is also a lifting of $f$. By the Lefschetz type theorem [12, Example 3.1.25], $\operatorname{Pic}(X)=$ $\left(\operatorname{Pic}\left(\mathbb{P}^{4}\right)\right) \mid X$.

For $V=V\left(E_{6}\right), V^{\prime}$ contains only one $(-1)$-curve $M^{\prime}$ and hence $V$ contains only one line $M$ (the image of $M^{\prime}$ ) by Lemma 2.3. Note that

$$
\{Q\}:=\operatorname{Sing} V \subset M \text {. }
$$

Let

$$
\Pi_{M M} \subset \mathbb{P}^{3}
$$

(say given by $X_{3}=0$ ) be the unique plane such that

$$
\Pi_{M M} \mid V=3 M
$$

Indeed, $3 M$ belongs to the complete linear system $|H| V \mid$, and the exact sequence

$$
0 \rightarrow \mathcal{O}(-2 H) \rightarrow \mathcal{O}(H) \rightarrow \mathcal{O}_{V}(H) \rightarrow 0
$$

and the vanishing of $H^{1}\left(\mathbb{P}^{3},-2 H\right)$ (e.g. by the Kodaira vanishing) imply

$$
H^{0}\left(\mathbb{P}^{3}, \mathcal{O}(H)\right) \simeq H^{0}\left(V, \mathcal{O}_{V}(H)\right) .
$$

Our $\pi^{*} \Pi_{M M}$ is a union of three 2-planes

$$
L_{i} \subset \mathbb{P}^{4}
$$

because the restriction of $\pi$ over $\Pi_{M M}$ is given by the equation

$$
Z^{3}=V\left(X_{0}, \ldots, X_{3}\right) \mid \Pi_{M M}=M\left(X_{0}, \ldots, X_{2}\right)^{3}
$$


where $M\left(X_{0}, \ldots, X_{2}\right)$ is a linear equation of $M \subset \Pi_{M M}$. This and the fact that $\pi^{*} \Pi_{M M}$ is a generator of $\operatorname{Pic}(X)=\left(\operatorname{Pic}\left(\mathbb{P}^{3}\right)\right) \mid X$, imply that the Weil divisor $L_{1}$ is not a Cartier divisor on $X$. Since $\operatorname{Sing} X$ consists of a single point $P$ lying over $\{Q\}=\operatorname{Sing} V, L_{1}$ is not Cartier at $P$ and hence $X$ is not factorial at $P$. Thus $X$ is not $\mathbb{Q}$-factorial at $P$ because the local $\pi_{1}$ of $P$ is trivial by a result of Milnor (cf. the proof of [10, Lemma 5.1]). Hence $g^{-1}(P)$ contains no smooth point (cf. [11, Lemma 5.16]) and must be equal to Sing $X=\{P\}$. Thus $f^{-1}(Q)=Q$ because $\pi^{-1}(Q)=P$.

2.8. Before we treat the case $V\left(3 A_{2}\right)$, we make some remarks. Up to isomorphism, there is only one $V\left(3 A_{2}\right)$ (cf. [17, Theorem 1.2]). Set $V:=V\left(3 A_{2}\right)$. There is a Gorenstein del Pezzo surface $W$ such that $\rho(W)=1$, Sing $W$ consists of four points $\beta_{i}$ of Du Val type $A_{2}$

$$
\pi_{1}(W \backslash \operatorname{Sing} W)=(\mathbb{Z} /(3))^{\oplus 2}
$$

and there is a Galois triple cover $V \rightarrow W$ étale over $W \backslash\left\{\beta_{1}, \beta_{2}, \beta_{3}\right\}$ so that a generator

$$
h \in \operatorname{Gal}(V / W)
$$

permutes the three singular points of $V$ lying over $\beta_{4}$ (cf. [13, Figure 1, Lemma 6]). Since the embedding $V \subset \mathbb{P}^{3}$ is given by the complete linear system $\left|-K_{V}\right|$ (cf. [9]) and $h^{*}\left(-K_{V}\right) \sim-K_{V}$, our $h$ extends to a projective transformation of $\mathbb{P}^{3}$, also denoted as $h$. Since $h(V)=V$,

$$
h^{*} V\left(X_{0}, \ldots, X_{3}\right)=c V\left(X_{0}, \ldots, X_{3}\right)
$$

for some nonzero constant $c$. This $h$ lifts to a projective transformation of $\mathbb{P}^{4}$, also denoted as $h$, stabilizing the above triple cover $X \subset \mathbb{P}^{4}$ of $\mathbb{P}^{3}$ by defining $h^{*} Z=\sqrt[3]{c} Z$. Then this $h$ permutes the three singular points of $X$ lying over $\operatorname{Sing} V$.

2.9. For $V=V\left(3 A_{2}\right), V^{\prime}$ has exactly three $(-1)$-curves $M_{i}^{\prime}$ and their images $M_{i}$ are therefore the only lines on $V$ (cf. Lemma 2.3). The graph $\sum M_{i}$ is triangle-shaped whose vertices (the intersection $M_{i} \cap M_{j}$ ) are the three points in Sing $V$. The sum of the three $(-1)$-curves $M_{i}^{\prime}$ and three (-2)-chains of type $A_{2}$ is linearly equivalent to $-K_{V^{\prime}}$ and hence $\sum M_{i} \sim-K_{V}$; also $2 M_{a} \sim M_{b}+M_{c}$ so long $\{a, b, c\}=\{1,2,3\}$; indeed, the three $M_{i}^{\prime}$ and the six (-2)-curves form the support of two singular fibres and two cross-sections of some $\mathbb{P}^{1}$-fibration. Thus $3 M_{i} \sim-K_{V} \sim H \mid V$. As argued in the case $V\left(E_{6}\right)$, there is a unique hyperplane $\Pi_{i}$ such that

$$
\Pi_{i} \mid V=3 M_{i}
$$

our $\pi^{*} \Pi_{i}$ is a union of three 2-planes $L_{i j}$ in $\mathbb{P}^{4}$ (sharing a line lying over $M_{i} \subset \mathbb{P}^{3}$ ), $L_{i 1}$ is not a Cartier divisor on $X$, the $X$ is not $\mathbb{Q}$-factorial at least at one of the two points (and hence at both points, since the above $h$ permutes $\operatorname{Sing} X$ ) in $L_{i 1} \cap \operatorname{Sing} X$ (lying 
over $\left.M_{i} \cap \operatorname{Sing} V\right)$, and $g^{-1}(\operatorname{Sing} X)=\operatorname{Sing} X$. Thus $f^{-1}(\operatorname{Sing} V)=\operatorname{Sing} V$. Hence $f^{-3}$ fixes each point in $\operatorname{Sing} V$.

This completes the proof of Remark 1.3 for normal cubic surfaces and hence the whole of Remark 1.3 .

Remark 2.10. The proof of Theorem 1.1 actually shows: if $f_{V}: V \rightarrow V$ is an endomorphism (not necessarily the restriction of some $\left.f: \mathbb{P}^{3} \rightarrow \mathbb{P}^{3}\right)$ of $\operatorname{deg}\left(f_{V}\right)>1$ of a Gorenstein normal del Pezzo surface with $K_{V}^{2}=3$ (i.e., a normal cubic surface), then $V$ is equal to $V_{1}$ or $V_{2}$ in Theorem 1.1 in suitable projective coordinates.

\section{REFERENCES}

[1] M. Abe and M. Furushima, On non-normal del Pezzo surfaces, Math. Nachr. 260 (2003), 3-13.

[2] A. Beauville, Endomorphisms of hypersurfaces and other manifolds, Internat. Math. Res. Notices 2001, no. 1, 53-58.

[3] J.-V. Briend, S. Cantat and M. Shishikura, Linearity of the exceptional set for maps of $\mathbb{P}_{k}(\mathbb{C})$, Math. Ann. 330 (2004), 39-43.

[4] D. Cerveau and A. Lins Neto, Hypersurfaces exceptionnelles des endomorphismes de $C P(n)$, Bol. Soc. Brasil. Mat. (N.S.) 31 (2000), no. 2, 155-161.

[5] T. -C. Dinh and N. Sibony, Equidistribution speed for endomorphisms of projective spaces, Math. Ann. 347 (2010), no. 3, 613-626.

[6] I. V. Dolgachev, Topics in Classical Algebraic Geometry. Part I, January 19, 2009, at: http://www.math.lsa.umich.edu/ idolga/topics1.pdf

[7] J. E. Fornaess and N. Sibony, Complex dynamics in higher dimension. I, Complex analytic methods in dynamical systems (Rio de Janeiro, 1992), pp. 201-231, Astérisque 222, Soc. Math. France, 1994.

[8] C. D. Hacon and J. McKernan, On Shokurov's rational connectedness conjecture, Duke Math. J. 138 (2007), no. 1, 119-136.

[9] F. Hidaka and K. Watanabe, Normal Gorenstein surfaces with ample anti-canonical divisor, Tokyo J. Math. 4 (1981), no. 2, 319-330.

[10] Y. Kawamata, Crepant blowing-ups of 3-dimensional canonical singularities and its application to degeneration of surfaces, Ann. of Math. 127 (1988), 93-163.

[11] J. Kollár and S. Mori, Birational geometry of algebraic varieties, Cambridge Tracts in Math. 134, Cambridge Univ. Press, 1998.

[12] R. Lazarsfeld, Positivity in algebraic geometry. I. Classical setting: line bundles and linear series. Ergebnisse der Mathematik und ihrer Grenzgebiete. 3. Folge. A Series of Modern Surveys in Mathematics, Vol. 48. Springer-Verlag, Berlin, 2004.

[13] M. Miyanishi and D. -Q. Zhang, Gorenstein log del Pezzo surfaces of rank one, J. Algebra, 118 (1988), 63-84.

[14] N. Nakayama, On complex normal projective surfaces admitting non-isomorphic surjective endomorphisms, Preprint 2 September 2008.

[15] N. Nakayama and D. -Q. Zhang, Polarized endomorphisms of complex normal varieties, Math. Ann. 346 (2010), no. 4, 991-1018; also: arXiv:0908.1688v1.

[16] M. Reid, Nonnormal del Pezzo surfaces, Publ. Res. Inst. Math. Sci. Kyoto Univ. 30 (1994), 695-727. 
[17] Q. Ye, On Gorenstein log del Pezzo surfaces, Japan. J. Math. 28 (2002), no. 1, 87-136.

[18] D. -Q. Zhang, Polarized endomorphisms of uniruled varieties (with an Appendix by Y. Fujimoto and N. Nakayama), Compos. Math. 146 (2010) 145 - 168.

[19] D. -Q. Zhang, Invariant hypersurfaces of endomorphisms of projective varieties, Preprint 2009.

Department of Mathematics, National University of Singapore

10 Lower Kent Ridge RoAd, Singapore 119076

E-mail address: matzdq@nus.edu.sg 